\title{
\& Research Square \\ Genetically Predicted Cardiac Troponin I Concentrations and Risk of Stroke and Atrial Fibrillation
}

\section{Dandan Liu}

The Third Affiliated Hospital of Soochow University

\section{Yue Deng}

The Third Affiliated Hospital of Soochow Univeristy

Jiao Wang

The Third Affiliated Hospital of Soochow University

\section{Yanan Chen}

The Third Affiliated Hospital of Soochow University

\section{Jian Yu}

The Third Affiliated Hospital of Soochow University

\section{Boyu Tan}

The Third Affiliated Hospital of Soochow University

\section{Mengmeng Wang ( $\nabla$ w935017050@163.com )}

The Third Affiliated Hospital of Soochow University https://orcid.org/0000-0002-9890-8900

\section{Research Article}

Keywords: Cardiac troponin I, stroke, mendelian randomization, atrial fibrillation.

Posted Date: July 12th, 2021

DOl: https://doi.org/10.21203/rs.3.rs-699688/v1

License: (9) This work is licensed under a Creative Commons Attribution 4.0 International License. Read Full License

Version of Record: A version of this preprint was published at Journal of Stroke and Cerebrovascular Diseases on March 1st, 2022. See the published version at https://doi.org/10.1016/j.jstrokecerebrovasdis.2021.106267. 


\section{Abstract}

Background. Observational studies have shown that elevated circulating cardiac troponin I (cTnl) concentrations were associated with higher risk of stroke and atrial fibrillation, but the causality remains unclear. Therefore, we conducted a two-sample mendelian randomization study to evaluate the causal effects of cTnl concentrations on the risk of stroke subtypes and atrial fibrillation.

Methods. The instrumental variables for circulating cTnl concentrations were selected from a genomewide association study meta-analysis of 48,115 European individuals. Applying a 2-sample mendelian randomization approach, we examined the associations of circulating cTnI concentrations with stroke ( 40,585 cases and 406,111 controls), ischemic stroke (34,217 cases and 406,111 controls), ischemic stroke subtypes (cardioembolic, large artery, small vessel stroke), intracerebral hemorrhage (1,545 cases and 1,481 controls) and atrial fibrillation (60,620 cases and 970,216 controls).

Results. Genetically predicted elevated circulating cTnl concentrations were associated with increased risk of cardioembolic stroke (odds ratio [OR], 1.80; 95\% confidence interval [CI], 1.20-2.68; $P=0.004$ ). However, no significant association was observed for cTnl concentrations with large artery stroke, small vessel stroke, total stroke, ischemic stroke and intracerebral hemorrhage. Additionally, we also found that elevated cTnl concentrations were associated with higher risk of atrial fibrillation $(\mathrm{OR}, 1.30 ; 95 \% \mathrm{Cl}, 1.10-$ $1.53 ; P=0.003)$.

Conclusions. This study provides evidence that genetically predicted circulating cTnl concentrations are causally associated with increased risk of cardioembolic stroke and atrial fibrillation.

\section{Introduction}

Stroke is a common cause of death and disability worldwide. Although many risk factors for stroke have been established, a substantial proportion of stroke risk factors remain unknown. ${ }^{[16]}$ Cardiac troponin I $(\mathrm{cTnl})$ is a structural protein released during myocardial injury and is widely used for the diagnosis of myocardial infarction. ${ }^{[12]}$ Elevated circulating cTnl concentrations have shown to be associated with a higher risk of cardiovascular disease and death in the general population. ${ }^{[2,11]}$ Some observational studies investigated the association between cTnl concentrations and the risk of stroke, and these studies yielded inconsistent results. ${ }^{[8,9,18,20,26,28]}$ Few studies investigated the association of cTnl concentrations with different ischemic stroke (IS) subtypes. Given that different IS subtypes has different pathologic mechanisms, it is necessary to further assess the effect of cTnl concentrations on different IS subtypes. Additionally, much of the available evidence for the causal association between cTnl concentrations and risk of stroke comes from observational studies, which may be affected by confounding and reverse causality. Thus, whether cTnl concentrations are causally associated with the risk of stroke remains unknown. 
Mendelian randomization (MR) is a genetic technique in which genetic variants are used as proxies for

exposure to examine the causal relationship between an exposure and a disease outcome. ${ }^{[7,22]}$ Because genetic variants are randomly allocated at conception and unlikely to be affected by disease in later life, MR can avoid the confounding and reverse causality in observational studies. Recently, a genome-wide association study (GWAS) has identified several single nucleotide polymorphisms (SNPs) that are independently associated with circulating cTnl concentrations in the general population, ${ }^{[17]}$ providing potential tools for MR. In this study, we aimed to use a two-sample MR to evaluate whether genetically predicted circulating cTnI concentrations are causally associated with the risk of stroke, IS, IS subtypes (large artery stroke [LAS], small vessel stroke [SVS] and cardioembolic stroke [CES]), intracerebral hemorrhage (ICH). Atrial fibrillation (AF) is an important risk factor of stroke. Given that elevated cTnI concentrations has also shown to be associated with an increased risk of AF. ${ }^{[21,27]}$ Therefore, the causal effects of cTnl concentrations on AF was also investigated in this MR study.

\section{Data Sources}

The public available GWAS summary statistics used in this study were summarized in Table 1. Because all data came from published GWASs, no additional ethical approval from an institutional review board was needed for this study. 
Table 1

Data sources used in this study

$\begin{array}{lll}\text { Phenotype } & \begin{array}{l}\text { Sample size (total or } \\ \text { cases/controls) }\end{array} & \text { Ancestry Sources }\end{array}$

Exposures

Cardiac troponin I 48,115 European HUNT and GS:SFHS ${ }^{[13]}$

concentrations

Outcomes

$\begin{array}{lll}\text { Any stroke } & \begin{array}{l}40,585 \text { cases/406,111 European } \\ \text { controls }\end{array} & \text { MEGASTROKE }\end{array}$

Ischemic stroke $\quad 34,217$ cases/406,111 European MEGASTROKE

controls Consortium ${ }^{[16]}$

Cardioembolic stroke $\quad 7,193$ cases/406,111 European MEGASTROKE

controls Consortium $^{[16]}$

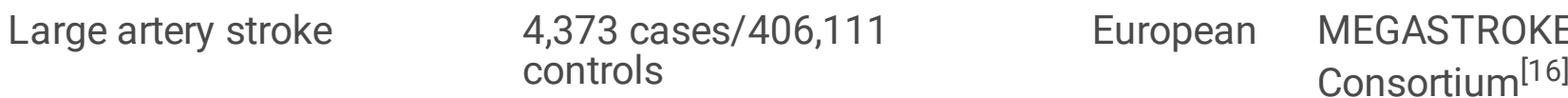

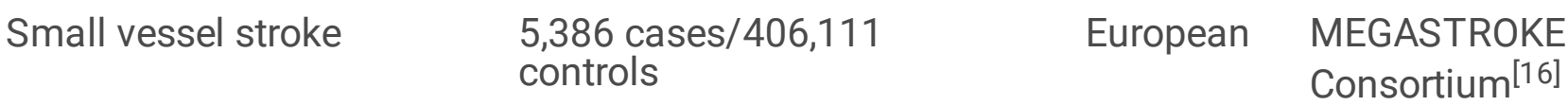

Intracerebral hemorrhage 1,545 cases/1,484 controls European ISGC meta-analysis ${ }^{[18]}$

Atrial fibrillation 60,620 cases/970,216 European GWAS meta-analysis of 6 controls studies $^{[19]}$

GWAS, genome-wide association study; HUNT, Trøndelag Health Study; GS:SFHS, Generation Scotland Scottish Family Health Study; ISGC: International Stroke Genetics Consortium.

\section{Genetic Instrument For Circulating Cardiac Troponin I Concentrations}

Instrumental variables for circulating cTnl concentrations were selected from a GWAS meta-analysis of the Trøndelag Health Study ((HUNT, $n=29,839)$ and the Generation Scotland Scottish Family Health Study (GS:SFHS, $n=18,276$ ) with 48,115 European individuals. ${ }^{[17]}$ This GWAS identified 12 genome-wide significant $\left(P<5 \times 10^{-8}\right)$ SNPs independently associated with cTnl concentrations (Supplementary Table 1). These 12 SNPs explained $1.2 \%$ phenotypic variation of circulating cTnI concentrations. ${ }^{[17]}$

\section{Outcome Data Sources}


Genetic association estimates for stroke, IS and IS subtypes were derived from the MEGASTROKE consortium. ${ }^{[14]}$ Briefly, we only used European GWAS summary statistics for stroke (40,585 cases and 406,111 controls), IS (34,217 cases and 406,111 controls) and IS subtypes (7,193 CES cases, 4,373 LAS cases, and 5,386 SVS cases). The IS subtypes were based on the the Trial of Org 10172 in Acute Stroke Treatment criteria (TOAST). ${ }^{[1]}$

For ICH, we used the summary statistics from the International Stroke Genetics Consortium (ISGC) metaanalysis including 1,545 cases and 1,481 controls. ${ }^{[24]}$

For AF, we used a GWAS meta-analysis of 6 studies (The Nord-Trøndelag Health Study, deCODE, the Michigan Genomics Initiative, DiscovEHR, UK Biobank, and the AFGen Consortium) including 60,620 cases and 970,216 controls of European ancestry. ${ }^{[19]}$

For the SNPs were not available in the outcome dataset, the proxy SNPs $(r 2>0.9)$ were used to replace them by using the LDlink (http:///dlink.nci.nih.gov) ${ }^{[13]}$ according to the CEU population data from 1,000 Genomes Project.

\section{Statistical analysis}

The primary MR analysis was performed by using inverse-variance weighted (IVW) method, ${ }^{[6]}$ which assumes that all genetic variants are valid genetic instruments (e.g., no directional pleiotropy). Given that the IVW estimate may be biased by the directional pleiotropy, weighted median ${ }^{[4]}$ and MR-Egger ${ }^{[3]}$ methods were also used to test the robustness of the IVW estimate. The weighted median method can provide a consistent estimate if at least $50 \%$ of the instrumental variables are valid. The MR-Egger approach can detect and adjust for the bias due to directional pleiotropy.

Several methods were conducted to evaluate the potential pleiotropy. First, we used Mendelian Randomization Pleiotropy Residual Sum and Outlier (MR-PRESSO) ${ }^{[23]}$ method to detect potential pleiotropic outlier $(P<0.1)$, and the IVW estimate was re-calculated if the pleiotropic outlier was identified. Second, the heterogeneity across different SNPs was assessed by using the Cochran's $Q$ statistics $(P<$ 0.05 indicates significant). Third, we performed MR-Egger intercept test to examine the directional pleiotropy, and the significant MR-Egger intercept test $(P<0.05)$ indicates the presence of directional pleiotropy. Finally, leave-one-out (LOO) analysis was used to test whether a single SNP drive the association.

Odds ratios (ORs) with $95 \%$ confidence intervals (95\% Cls) were scaled to 1- standard deviation (SD) increment of inverse rank transformed circulating cTnl concentrations. A Bonferroni corrected $P$ value $<$ 0.007 (correcting for 7 outcomes) was considered as statistically significant. All statistical analyses were performed using the Mendelian randomization, ${ }^{[25]}$ TwoSampleMR, ${ }^{[10]}$ and MR-PRESSO packages ${ }^{[23]}$ for R version 4.0.2. 


\section{Results}

\section{Genetically predicted circulating cTnl concentrations and risk of stroke}

A SNP rs151313792 for cTnl concentrations was not available in the stroke (total stroke, IS, IS subtypes) outcome dataset, and no proxy SNP $\left(r^{2}>0.8\right)$ can be used to replace it. The SNP rs8039472 was used as a proxy for rs $8024538\left(r^{2}=0.92\right)$, because rs 8024358 was not available in the stroke ((total stroke, IS, IS subtypes and $\mathrm{ICH}$ ) outcome dataset. For $\mathrm{ICH}$, a total of 4 SNPs were not available and no proxy SNP. Additionally, MR-PRESSO identified a outlier for CES outcome (Supplementary Table 2), and no outlier was identified for other stroke outcome (LAS, SVS, stroke, IS and ICH).

As shown in Fig. 1, after remove the outlier, the IVW estimates indicated that genetically predicted elevated cTnl concentrations were associated with an increased risk of CES $(\mathrm{OR}, 1.80 ; 95 \% \mathrm{Cl}, 1.20-2.68$; $P=0.004$; Fig. 1). Similar effect estimates were observed in weighted median and MR-Egger estimates, although with broader Cls due to lower statistical power (Fig. 1). The Cochran's $Q$ test suggested that no significant heterogeneity across these 10 SNPs (Cochran's $Q=16.91, P=0.050$; Supplementary Table 2). Similarly, there was no evidence of directional pleiotropy (MR-Egger intercept, $P=0.991$; Supplementary Table 2). The LOO analysis indicated that no single SNP drove the significant association (Supplementary Fig. 1).

Conversely, IVW analysis suggested that genetically predicted elevated cTnl concentrations were not associated with LAS, SVS, total stroke, IS, and ICH (Fig. 1). The Cochran's $Q$ test indicated that no significant heterogeneity ( $P>0.160$; Supplementary Table 2$)$, and there was no evidence of directional pleiotropy (MR-Egger intercept, $P>0.320$; Supplementary Table 2). Similarly, The LOO analysis suggested that no single SNP drove the results (Supplementary Fig. 2-6).

\section{Genetically predicted circulating cTnl concentrations and risk of atrial fibrillation}

For AF, MR-PRESSO identified 3 SNP outliers (Supplementary Table 2). After removing these 3 outliers, genetically predicted elevated cTnl concentrations were associated with an increased risk of AF (IVW OR, $1.30 ; 95 \% \mathrm{Cl}, 1.10-1.53 ; P=0.003 ; \mathrm{Fig} .1)$. Weighted median and MR-Egger estimates yield similar causal effect estimates. The Cochran's $Q$ test indicated that significant heterogeneity across individual SNPs (Cochran's $Q=18.97, P=0.015$; Supplementary Table 2), suggesting that the presence of horizontal pleiotropy. However, the MR-Egger intercept test found no evidence of directional pleiotropy (MR-Egger intercept, $P=0.614$; Supplementary Table 2), indicating that the horizontal pleiotropy was unlikely to bias the IVW estimate. Additionally, the LOO analysis did not find any single SNP drove the significant association (Supplementary Fig. 7).

\section{Discussion}


To the best of our knowledge, this was the first MR study to investigate the relationship between circulating cTnI concentrations and risk of stroke, IS, IS subtypes, ICH and AF using a two-sample MR approach. Our findings indicate that genetically predicted elevated cTnl concentrations were causally associated with an increased risk of CES but not LAS, SVS, stroke, ischemic stroke and ICH. Additionally, we also found that elevated cTnl concentrations were associated with higher risk of AF.

Recently, a meta-analysis of 12 studies $^{[5]}$ performed in general population indicated that elevated highsensitivity assayed troponins is associated with an increased risk of stroke. However, this meta-analysis has a high heterogeneity, and stroke subtypes were not provided in this study. ${ }^{[5]}$ Another study ${ }^{[8]}$ conducted in 9 European community-based cohorts demonstrated that elevated hsTnl concentrations were associated with increased risk of stroke, IS and ICH. However, these studies are observational studies that are easily influenced by its inherent limitation, such as confounding and reverse bias. Therefore, in order to provide causal evidence for the effects of cTnl concentrations on the risk of stroke, we conducted this MR study to reduce the possibility of confounding and reverse causation. In this MR study, we found that circulating cTnl concentrations were only associated with increased risk of CES but not with LAS, SVS, total stroke, IS and ICH. A possible explanation for the observed significant association between CTnl concentrations and CES is that elevated cTnl concentrations may be related with asymptomatic arrhythmias, in particular, AF. ${ }^{[15]}$ Several previous studies have reported that elevated cTnl concentrations were associated with an increased risk of $A F$ in the general population. ${ }^{[21,27]}$ Hence, using the MR approach, we also evaluate the association of genetically predicted cTnl concentrations with $A F$, and found that genetically predicted cTnl concentrations were causally associated with an increased risk of AF. Therefore, the observed association of cTnl concentrations with CES might be related to AF. Further studies are still needed to investigate the mechanisms by which elevated cTnI concentrations increased the risk of CES.

The major strength of this study is the two-sample MR study design, which can reduce the confounding factors and reverse causality in observational studies. Other important strengths are the large samples of stroke cases and that associations with IS subtypes can be further investigated. However, several limitations still needed to be noticed. The major limitation of MR study is potential pleiotropy, which may bias the IVW estimates. Therefore, several approaches were conducted to evaluate and adjust for potential pleiotropy. The potential outliers identified by MR-PRESSO method were excluded before IVW estimates. Weighed median and MR-Egger estimates that are relatively robust to pleiotropy, were also performed. LOO analysis and forest-plot were also used to evaluate whether a single SNP drive the association. The results of these analyses were consistent, indicating that this observed association was unlikely due to the pleiotropy. Additionally, our results were based on the samples of European ancestry. Thus, these findings in this study might not be extrapolated to other population.

To conclude, this MR study provided evidence to support that genetically predicted elevated cTnI concentrations are causally associated with increased risk of CES and AF. 


\section{Declarations}

Acknowledgements

All MEGASTROKE consortium authors are listed in Supplementary material. The MEGASTROKE project received founding from source specified at https://www.megastroke.org/acknowledgements.html.

Funding: None

\section{Data availability statement}

All the data were from original publicly available GWAS summary statistics (Please see Table 1).

Ethics Approval All relevant ethics approval are from original GWASs.

Consent to Participate This study only used publicly available summary statistics from published GWASs. No additional informed consent were needed in this study.

Consent for Publication No individual-level data were involved, and no additional consent for publication was needed for this study.

\section{Conflict of interest}

None

\section{Author contributions}

Dandan Liu, Yue Deng and Mengmeng Wang conceived and designed the study; Dandan Liu, Jiao Wang and Mengmeng Wang conducted the statistical analysis and drafted the manuscript; Dandan Liu, Yanan Chen acquired the data; Yue Deng, Jian Yu, Boyu Tan and Mengmeng Wang revised the manuscript.

\section{References}

1. Adams HP, Bendixen BH, Kappelle LJ, Biller J, Love BB, Gordon DL, Marsh EE (1993) Classification of subtype of acute ischemic stroke. Definitions for use in a multicenter clinical trial. TOAST. Trial of Org 10172 in Acute Stroke Treatment. Stroke 24:35-41

2. Blankenberg S, Salomaa V, Makarova N, Ojeda F, Wild P, Lackner KJ, Jørgensen T, Thorand B, Peters A, Nauck M, Petersmann A, Vartiainen E, Veronesi G, Brambilla P, Costanzo S, lacoviello L, Linden G, Yarnell J, Patterson CC, Everett BM, Ridker PM, Kontto J, Schnabel RB, Koenig W, Kee F, Zeller T, Kuulasmaa K (2016) Troponin I and cardiovascular risk prediction in the general population: the BiomarCaRE consortium. Eur Heart J 37:2428-2437

3. Bowden J, Davey Smith G, Burgess S (2015) Mendelian randomization with invalid instruments: effect estimation and bias detection through Egger regression. Int J Epidemiol 44:512-525 
4. Bowden J, Davey Smith G, Haycock PC, Burgess S (2016) Consistent Estimation in Mendelian Randomization with Some Invalid Instruments Using a Weighted Median Estimator. Genet Epidemiol 40:304-314

5. Broersen LHA, Stengl H, Nolte CH, Westermann D, Endres M, Siegerink B, Scheitz JF (2020) Association Between High-Sensitivity Cardiac Troponin and Risk of Stroke in 96702 Individuals: A Meta-Analysis. Stroke 51:1085-1093

6. Burgess S, Bowden J, Fall T, Ingelsson E, Thompson SG (2017) Sensitivity Analyses for Robust Causal Inference from Mendelian Randomization Analyses with Multiple Genetic Variants. Epidemiology 28:30-42

7. Burgess S, Thompson SG (2015) Mendelian Randomization: Methods for Using Genetic Variants in Causal Estimation. Chapman \& Hall/CRC Press, London

8. Camen S, Palosaari T, Reinikainen J, Sprünker NA, Niiranen T, Gianfagna F, Vishram-Nielsen JKK, Costanzo S, Söderberg S, Palmieri L, Ferrario M, Peters A, Vartiainen E, Donati MB, Donfrancesco C, Borchini R, Börschel CS, Giampaoli S, Di Castelnuovo A, Magnussen C, Kee F, Koenig W, Blankenberg S, de Gaetano G, Tunstall-Pedoe H, Rospleszcz S, Jørgensen T, Zeller T, Kuulasmaa K, Linneberg A, Salomaa V, lacoviello L, Schnabel RB (2020) Cardiac Troponin I and Incident Stroke in European Cohorts: Insights From the BiomarCaRE Project. Stroke 51:2770-2777

9. Everett BM, Zeller T, Glynn RJ, Ridker PM, Blankenberg S (2015) High-sensitivity cardiac troponin I and B-type natriuretic Peptide as predictors of vascular events in primary prevention: impact of statin therapy. Circulation 131:1851-1860

10. Hemani G, Zheng J, Elsworth B, Wade KH, Haberland V, Baird D, Laurin C, Burgess S, Bowden J, Langdon R, Tan VY, Yarmolinsky J, Shihab HA, Timpson NJ, Evans DM, Relton C, Martin RM, Davey Smith G, Gaunt TR, Haycock PC (2018) The MR-Base platform supports systematic causal inference across the human phenome. Elife 7

11. Jia X, Sun W, Hoogeveen RC, Nambi V, Matsushita K, Folsom AR, Heiss G, Couper DJ, Solomon SD, Boerwinkle E, Shah A, Selvin E, de Lemos JA, Ballantyne CM (2019) High-Sensitivity Troponin I and Incident Coronary Events, Stroke, Heart Failure Hospitalization, and Mortality in the ARIC Study. Circulation 139:2642-2653

12. Keller T, Zeller T, Peetz D, Tzikas S, Roth A, Czyz E, Bickel C, Baldus S, Warnholtz A, Fröhlich M, Sinning CR, Eleftheriadis MS, Wild PS, Schnabel RB, Lubos E, Jachmann N, Genth-Zotz S, Post F, Nicaud V, Tiret L, Lackner KJ, Münzel TF, Blankenberg S (2009) Sensitive troponin I assay in early diagnosis of acute myocardial infarction. N Engl J Med 361:868-877

13. Machiela MJ, Chanock SJ (2015) LDlink: a web-based application for exploring population-specific haplotype structure and linking correlated alleles of possible functional variants. Bioinformatics 31:3555-3557

14. 14

15. McCarthy CP, Yousuf O, Alonso A, Selvin E, Calkins H, McEvoy JW (2017) High-Sensitivity Troponin as a Biomarker in Heart Rhythm Disease. Am J Cardiol 119:1407-1413 
16. Meschia JF, Bushnell C, Boden-Albala B, Braun LT, Bravata DM, Chaturvedi S, Creager MA, Eckel RH, Elkind MSV, Fornage M, Goldstein LB, Greenberg SM, Horvath SE, ladecola C, Jauch EC, Moore WS, Wilson JA (2014) Guidelines for the primary prevention of stroke: a statement for healthcare professionals from the American Heart Association/American Stroke Association. Stroke 45:37543832

17. Moksnes MR, Røsjø H, Richmond A, Lyngbakken MN, Graham SE, Hansen AF, Wolford BN, Gagliano Taliun SA, LeFaive J, Rasheed H, Thomas LF, Zhou W, Aung N, Surakka I, Douville NJ, Campbell A, Porteous DJ, Petersen SE, Munroe PB, Welsh P, Sattar N, Smith GD, Fritsche LG, Nielsen JB, Åsvold BO, Hveem K, Hayward C, Willer CJ, Brumpton BM, Omland T (2021) Genome-wide association study of cardiac troponin I in the general population. Hum Mol Genet

18. Neumann JT, Havulinna AS, Zeller T, Appelbaum S, Kunnas T, Nikkari S, Jousilahti P, Blankenberg S, Sydow K, Salomaa V (2014) Comparison of three troponins as predictors of future cardiovascular events-prospective results from the FINRISK and BiomaCaRE studies. PLoS ONE 9:e90063

19. Nielsen JB, Thorolfsdottir RB, Fritsche LG, Zhou W, Skov MW, Graham SE, Herron TJ, McCarthy S, Schmidt EM, Sveinbjornsson G, Surakka I, Mathis MR, Yamazaki M, Crawford RD, Gabrielsen ME, Skogholt AH, Holmen OL, Lin M, Wolford BN, Dey R, Dalen H, Sulem P, Chung JH, Backman JD, Arnar DO, Thorsteinsdottir U, Baras A, O'Dushlaine C, Holst AG, Wen X, Hornsby W, Dewey FE, Boehnke M, Kheterpal S, Mukherjee B, Lee S, Kang HM, Holm H, Kitzman J, Shavit JA, Jalife J, Brummett CM, Teslovich TM, Carey DJ, Gudbjartsson DF, Stefansson K, Abecasis GR, Hveem K, Willer CJ (2018) Biobank-driven genomic discovery yields new insight into atrial fibrillation biology. Nat Genet 50:1234-1239

20. Nylander R, Lind L, Wikström J, Lindahl B, Venge P, Larsson A, Ärnlöv J, Berglund L, Ahlström H, Johansson L, Larsson E-M (2015) Relation between cardiovascular disease risk markers and brain infarcts detected by magnetic resonance imaging in an elderly population. J Stroke Cerebrovasc Dis 24:312-318

21. Rienstra M, Yin X, Larson MG, Fontes JD, Magnani JW, McManus DD, McCabe EL, Coglianese EE, Amponsah M, Ho JE, Januzzi JL, Wollert KC, Fradley MG, Vasan RS, Ellinor PT, Wang TJ, Benjamin EJ (2014) Relation between soluble ST2, growth differentiation factor-15, and high-sensitivity troponin I and incident atrial fibrillation. Am Heart J 167

22. Smith GD, Ebrahim S (2003) 'Mendelian randomization': can genetic epidemiology contribute to understanding environmental determinants of disease? Int J Epidemiol 32

23. Verbanck M, Chen C-Y, Neale B, Do R (2018) Detection of widespread horizontal pleiotropy in causal relationships inferred from Mendelian randomization between complex traits and diseases. Nat Genet 50:693-698

24. Woo D, Falcone GJ, Devan WJ, Brown WM, Biffi A, Howard TD, Anderson CD, Brouwers HB, Valant V, Battey TWK, Radmanesh F, Raffeld MR, Baedorf-Kassis S, Deka R, Woo JG, Martin LJ, Haverbusch M, Moomaw CJ, Sun G, Broderick JP, Flaherty ML, Martini SR, Kleindorfer DO, Kissela B, Comeau ME, Jagiella JM, Schmidt H, Freudenberger P, Pichler A, Enzinger C, Hansen BM, Norrving B, JimenezConde J, Giralt-Steinhauer E, Elosua R, Cuadrado-Godia E, Soriano C, Roquer J, Kraft P, Ayres AM, 
Schwab K, McCauley JL, Pera J, Urbanik A, Rost NS, Goldstein JN, Viswanathan A, Stögerer E-M, Tirschwell DL, Selim M, Brown DL, Silliman SL, Worrall BB, Meschia JF, Kidwell CS, Montaner J, Fernandez-Cadenas I, Delgado P, Malik R, Dichgans M, Greenberg SM, Rothwell PM, Lindgren A, Slowik A, Schmidt R, Langefeld CD, Rosand J (2014) Meta-analysis of genome-wide association studies identifies 1q22 as a susceptibility locus for intracerebral hemorrhage. Am J Hum Genet 94:511-521

25. Yavorska 00, Burgess S (2017) MendelianRandomization: an R package for performing Mendelian randomization analyses using summarized data. Int $\mathrm{J}$ Epidemiol 46:1734-1739

26. Zeller T, Tunstall-Pedoe H, Saarela O, Ojeda F, Schnabel RB, Tuovinen T, Woodward M, Struthers A, Hughes M, Kee F, Salomaa V, Kuulasmaa K, Blankenberg S (2014) High population prevalence of cardiac troponin I measured by a high-sensitivity assay and cardiovascular risk estimation: the MORGAM Biomarker Project Scottish Cohort. Eur Heart J 35:271-281

27. Zhu K, Hung J, Divitini M, Murray K, Lim EM, St John A, Walsh JP, Knuiman M (2018) High-sensitivity cardiac troponin I and risk of incident atrial fibrillation hospitalisation in an Australian communitybased cohort: The Busselton health study. Clin Biochem 58:20-25

28. Zhu K, Knuiman M, Divitini M, Murray K, Lim EM, St John A, Walsh JP, Hung J (2018) High-sensitivity cardiac troponin I and risk of cardiovascular disease in an Australianpopulation-based cohort. Heart 104:895-903

\section{Figures}


Figure 1

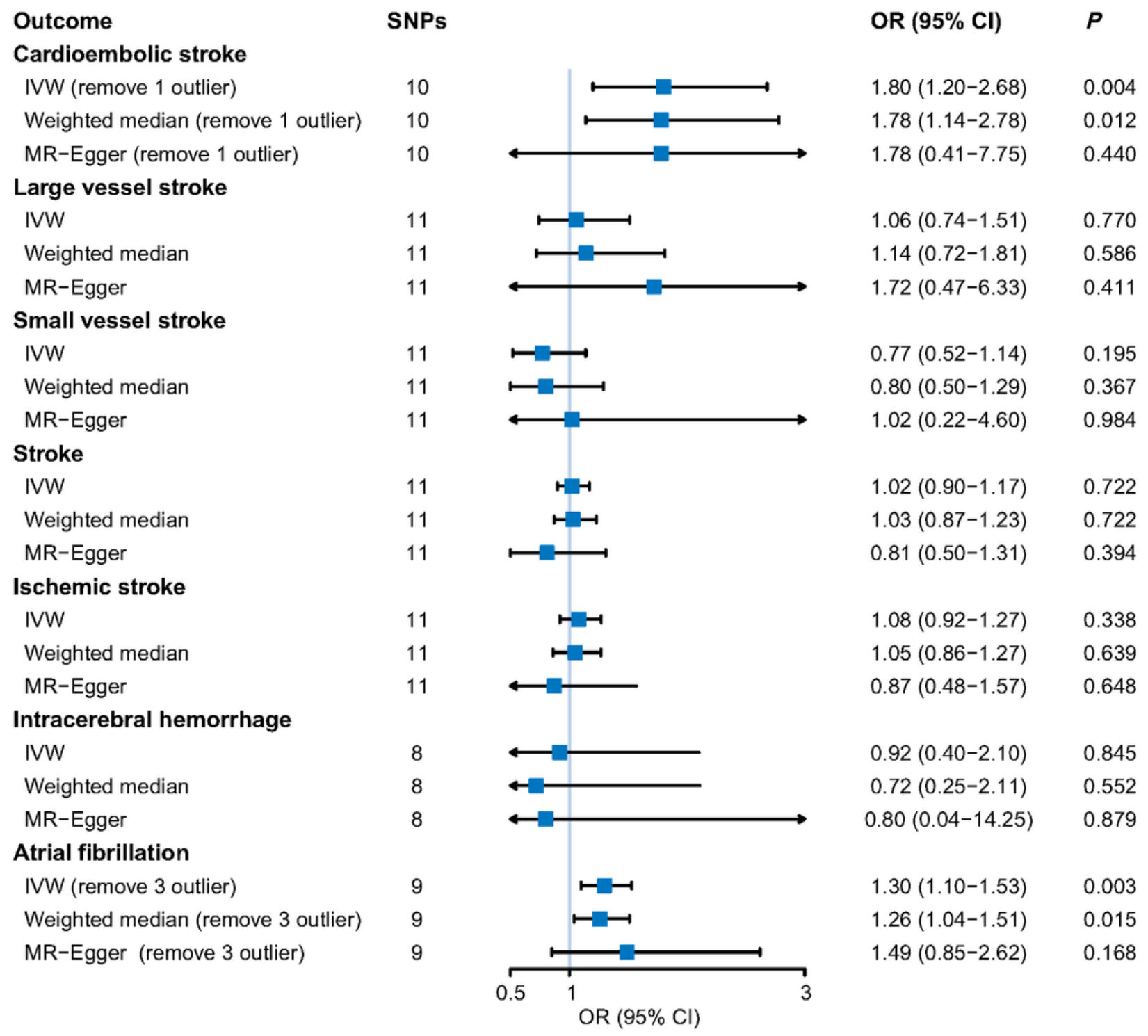

\section{Figure 1}

Mendelian randomization estimates of circulating cardiac troponin I concentrations and risk of stroke and atrial fibrillation.

\section{Supplementary Files}

This is a list of supplementary files associated with this preprint. Click to download.

- Supplementarymeterial.pdf 